\title{
A Morphological Methodology for Features Identification in Satellite Images for Semi-automatic Cartographic Updating
}

\author{
Michele Mengucci and Fernando Muge \\ CRVM Centro de Geo-Sistemas do I.S.T. \\ Av.Rovisco Pais 1 \\ 1049-001 Lisboa, Portugal \\ Tel. +351218417247-Fax +351218417442 \\ mmengucci@ist.utl.pt
}

\begin{abstract}
This work investigates a way to exploit the information of satellite images in order to identify cartographic features, aiming at developing a software tool able to update digital maps automatically. A cartographic feature, like any object present in a multi-channel image, is a set of pixels with similar spectral response and a certain spatial relation between them. The current algorithm works iteratively and mixes the spatial information with the spectral one in an appropriate way to finally detect the whole shape of a cartographic feature starting from a pixel marked previously by the user in a remotely sensed image. It is also shown that Mathematical Morphology (MM) operators can handle the spatial and spectral information decreasing the computational cost. First, the structure of the main algorithm is presented, showing each step of its operational sequence. Then, some application examples are reported and, finally, some remarks illustrate the future possibilities of implementation and development of the algorithm.
\end{abstract}

\section{Introduction}

Objects in multi-channel images, particularly remotely sensed ones, are set of pixels that can be assembled together by their individual spectral response and by their neighborhood relationship in the spatial domain of each channel. Although there exist in the literature several works that propose to control the segmentation through spectral information [1] [2] [3], there is not a general procedure that combines both types of information. Thus, in this paper, we introduce a novel methodology, whose main objective consists of generating objects by assembling together small elementary regions that are simultaneously spectral and spatial neighbors. Usually the classification procedures are distinguished between boundary seeking and region growing [4] [5], whether the objects are defined detecting their edge pixels, or as regions in which the pixel values are homogeneous. The proposed algorithm firstly divides the image's spatial domain in small areas and then applies a region growing process to the areas instead than directly to the pixels, so the spectral information is processed in two steps, on the pixel scale and on the elementary region scale. 
The first partitioning is performed with a watershed [6] transformation on the spectral gradient [7] [8] [9], thus obtaining a fine segmentation that preserves the image spectral meaning. Therefore a unique and representative spectral value can be assigned to each area, producing a multispectral mosaic image [6]. On the latter a region growing procedure performs an iterative merging of the regions with similar spectral value, starting from the areas marked by the user. The user controls the similarity between regions by a spectral distance parameter previously defined. The region merging process is obviously much faster than a pixel merging one, even considering the computational time of the preliminary watershed. Apart from this, objects created with a fixed shape kernel on each pixel usually have a border that reflects this geometry while this procedure maintains the details of the objects morphology as perceived by human vision.

\section{Fusion Algorithm}

This name recalls the merging which is the main operation of this methodology. This algorithm takes the pieces of the mosaic (the basins of the watershed) that are spatial neighbors, and merges them into together into a new one.

This idea is meant for any n-dimensional multi-channel image but this article illustrates it in the bi-dimensional case, on a simple set of two images. This simplification maintains the validity of the multi-spectral aspect and is convenient because it worked with less computing expense and it allows a graphical description of the process: indeed we can see the projection of mosaic pieces as points in a two dimensional image, where their coordinates are their grey level in each band, moreover it allows a graphical representation of the concepts of spatial and spectral proximity, which is difficult in a case of a three-dimensional set, and impossible with more dimensions. Finally the bi-dimensionality lets us run the whole algorithm, even when it approaches the multispectral data, in terms of MM transforms, as the distances calculations in the spectral space are performed by the binary dilation.

\subsection{Mosaic Images}

A quick remark on the basic support for this procedure has to be done before describing in details the steps of the algorithm. The mosaic image to be used is slightly different from the ones found in the literature [6]. Generally a mosaic image is created from a grey level image once this is divided in areas and a unique grey value is assigned to each one calculated on the values of its pixels. There are different mosaic versions that differ in the value assigned to object and to the boundary between the areas. Many versions [7] [12] have been used but the one presented here, has two main characteristics: first, the objects are obtained applying the watershed on the multi-spectral gradient, in order to get a detailed separation in catchment basins with homogeneous spectral meaning; second, the watershed line between the adjacent basins has value zero, in order to keep them separated. The binary image with the partition in small areas, viewed as "pieces of the mosaic", is here called as "pre-mosaic" (Fig.1b). 


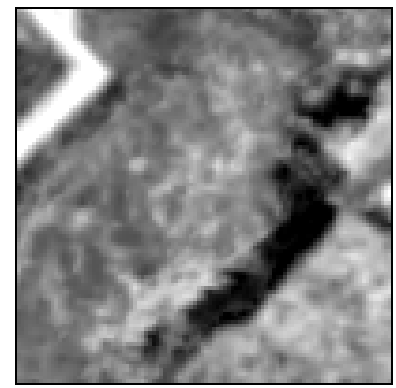

a

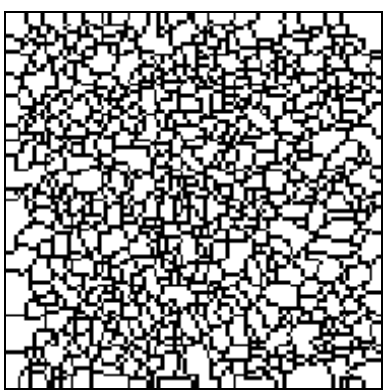

b

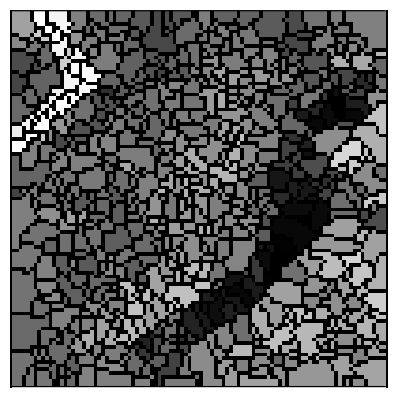

c

Fig. 1. Original image (a), catchment basins or "pre_mosaic" (b) and mosaic image (c).

\subsection{Steps of the Algorithm}

\section{Inputs}

First, the set of images must be transformed into mosaics. In this case we have two mosaics that were selected from a set of five images. Then a marker image is created. It can be obtained manually, marking some points belonging to the requested object, or automatically, if at least part of the object can be detected in this way. Basically this image should indicate the pieces that belong "a priori" to the required object (Fig.2).

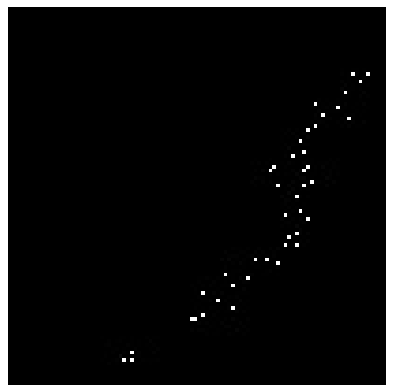

a

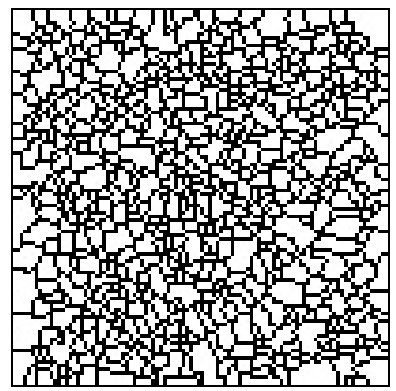

b

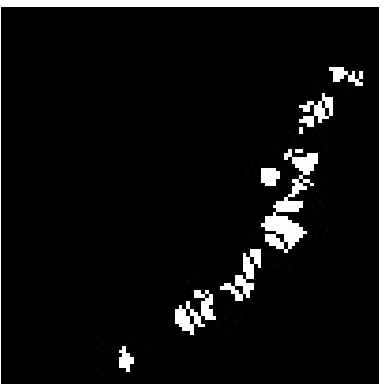

c

Fig. 2. Reconstruction (c) of the pre-mosaic image (b) by the marker image (a).

Step 1: Reconstruction of a marker image in the pre mosaic image: only the pieces of the mosaic hit by the marker image (intersection not empty) are considered from the "pre-mosaic" (Fig. 2c).

Step 2: The algorithm considers one by one all the pieces marked in the previous step, and for each one of them finds the pieces that are spatial neighbors (Fig. 3b).

Step 3: The spectral values of a piece, and its spatial neighbors, are visualized as points in the spectral space (Fig. 4a, 4b). 


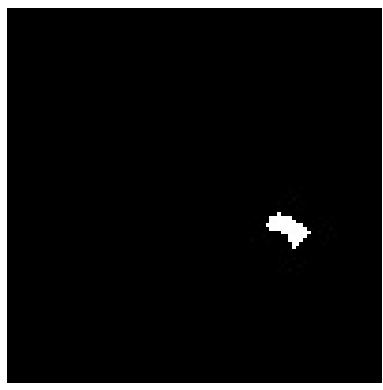

$\mathbf{a}$

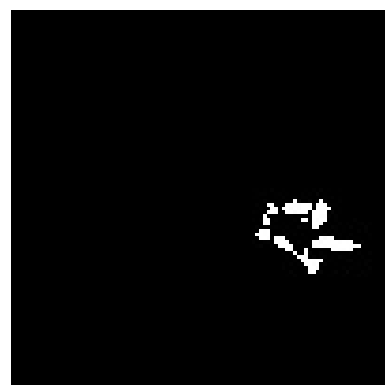

b

Fig. 3. Spatial domain: single piece (a); spatial neighbors (b).

Step 4: With an intersection between a dilation of the projection of the piece under evaluation and the projections of its spatial neighbors, only the projections of the spatial and spectral neighbors are selected. The size of the dilation is the key parameter of this process and it can be different in each channel depending on the contrast relation between the object and the background grey values, which usually varies from one channel to the other. In this case (Fig. 4c) it would mean that the dilation could have different values in the horizontal and the vertical direction.

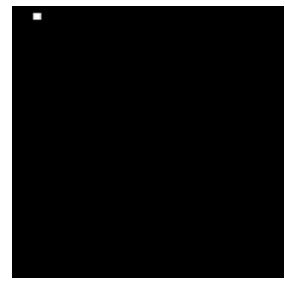

$\mathbf{a}$

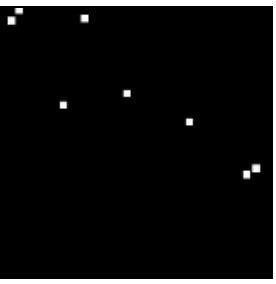

b

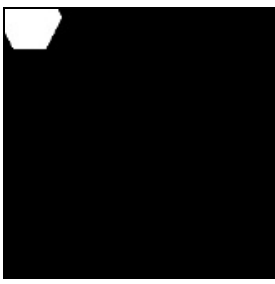

c

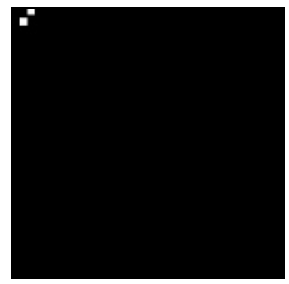

d

Fig. 4. Spectral domain: projections of the single piece in fig. 3a (a); projection of its spatial neighbors (shown in fig. 3b) (b); dilation of the single piece projection (c); intersection to identify the spectral neighbors that are already spatial neighbors (d).

Step 5: Knowing the spectral coordinates of the projections of the spatial and spectral neighbors the algorithm returns to the initial images to identify them (Fig. 5a) and merges them with the initial piece into a new bigger one (Fig. 5b).

Step 6: Each piece grows to a larger shape that is the result of the merging procedure. Gradually the new merged pieces modify the mosaics, and update the input images for the next loop of the application. The new pieces of the mosaic images have a grey value that is an average of the grey levels of the merged pieces, weighted on their areas.

These six steps are applied on each previously marked piece and the whole process is looped on all the pieces until the algorithm can find no more spectral neighbors. 


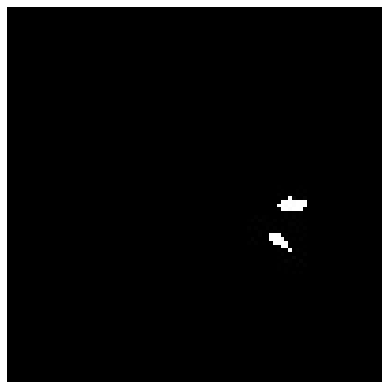

$\mathbf{a}$

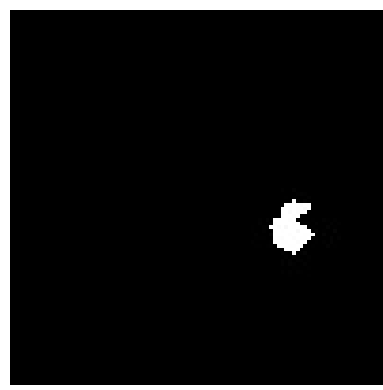

b

Fig. 5. Spatial domain: identification of the spatial neighbors that are spectral neighbors (a); merging procedure (b).

\section{Case Studies}

The developed methodology and algorithms were applied to several remotely sensed images; in particular it has been experimented to detect cartographic features in SPOT satellite images over the area of Luanda (Angola). We present in this paper some examples concerning different cartographic layers. The first one concerns the study of a river (Fig.6a), which is correctly identified (Fig.6b). The contours (Fig.6c) and the medial axis (skeleton) (Fig.6d) of the river, superimposed to the initial image, testify the success of application of our approach. This example also gives a hint on how to use the algorithm results in updating maps, for example this would be the first step towards creating a hydrology GIS layer.

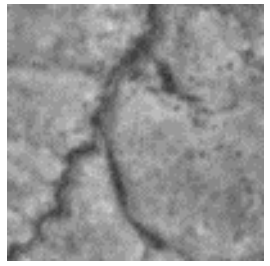

a

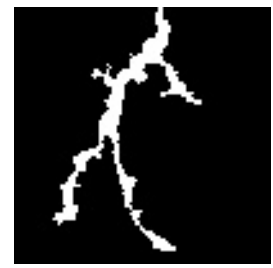

b

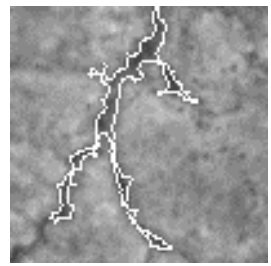

c

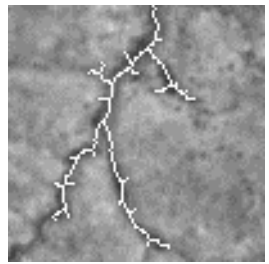

d

Fig. 6. Single channel of cut to object in the original image (a), algorithm detected shape (b), boundaries (c) and skeleton of the object (d).

Using old cartography features as markers to new ones should theoretically work well. Indeed many human built cartographic features (roads, urban areas) tend to expand over the years so the old are included in the recent objects but this procedure can present some drawbacks. For example in Fig.7, because of its slight displacement due to the incorrect registration between the map and the satellite images, the old route could not be used as a marker and forced the use manual markers. 


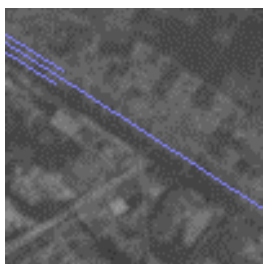

$\mathbf{a}$

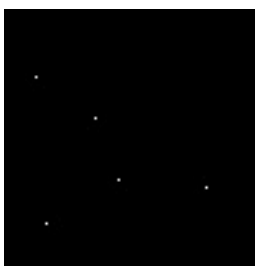

b

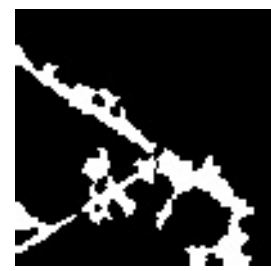

c

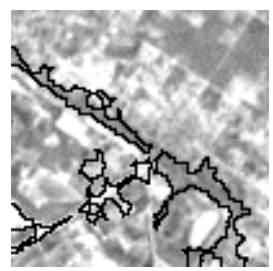

d

Fig. 7. Cut of sub-urban area with old cartography overlaid (a), user made markers (b), algorithm detected shape (c) and boundaries on single channel (d).

The algorithm proved its efficiency in the case of the airport, another object considered relevant as an object to detect. In Fig.8 two input mosaic images are exposed to illustrate the different information between the channels of the remote sensed images and the way that shapes are highlighted and its gray levels simplified. The input channels are the XS3 and XS1, corresponding to the Blue and the Red bands of the SPOT, and they cover almost all the territory occupied by the city of Luanda airport. The marker image is very simple; it has just 4 pixels marked, and has been created specially to extract the shape of the airstrips. As shown in the figure these points were enough to reconstruct the whole shape.
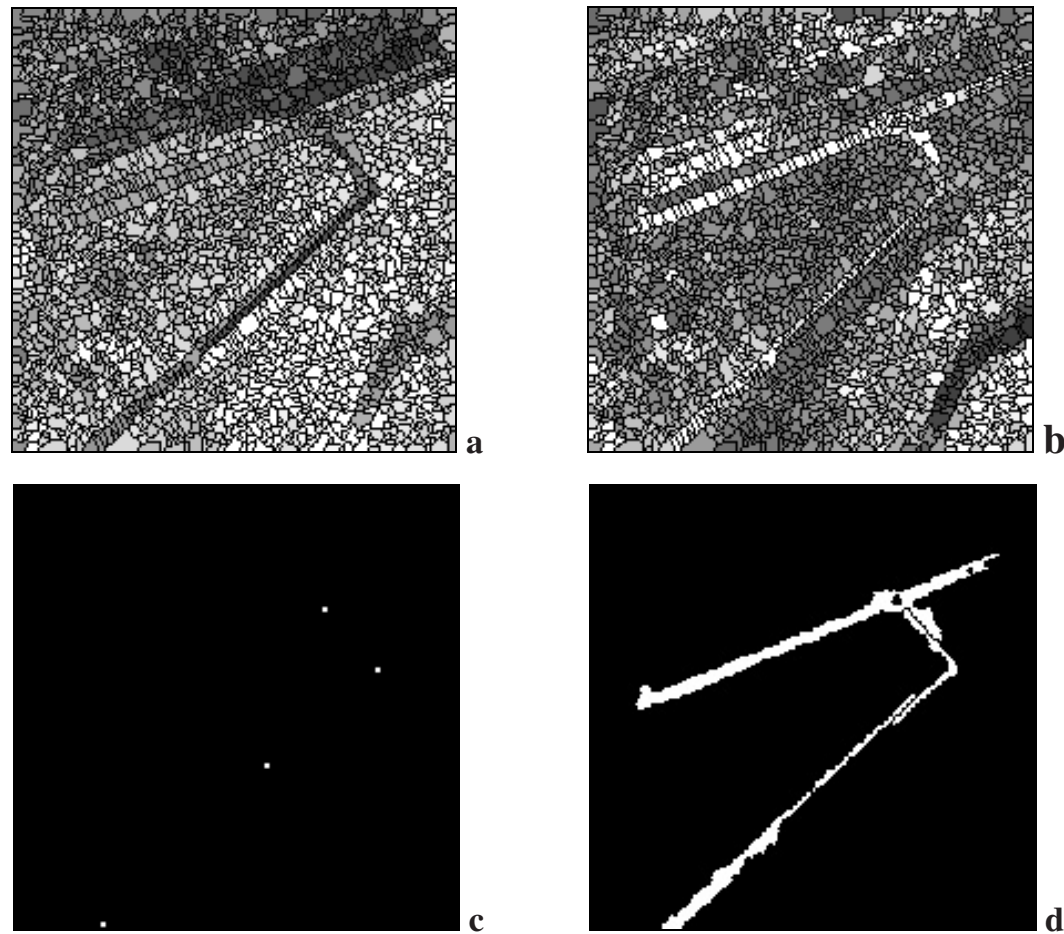

Fig. 8. Input mosaics (a and b), marker image (c) and resulting object (d). 


\section{Conclusion and Future Researches}

This methodology belongs to the "region growing" procedures' class but its distinguishing feature is that the elementary regions to be merged are not pixels but presegmented "textural elementary units" [13]. The pieces of the mosaic images, obtained with MM operators, can be represented as points in the spectral features space. For this, the region growing procedure becomes much faster and the objects shape found suites better to their morphology as perceived by human vision.

It gives good and useful results and it is actually a good tool to find connected shapes with a spectral meaning, when applied to remotely sensed images. It is not in its definitive version and it is still liable to be modified to improve its efficiency. The future improvements will be done towards a better interaction with users, and to test and compare different modifications to the structure described in 2.2.

Apart from the marker image the algorithm has another aspect where the user interaction is quite important: the spectral distance (2.2 Step 4). This is the parameter that decides whether the projections of the pieces in the spectral space are neighbors or not. It is a distance parameter that has a component in each band. In the future modifications it will be improved the semi-automatic definition of these. The user will be requested to make some click on the object (object clicks) in the image and some in the background, in points that do not belong to the object "a priori" (background clicks). In this way the algorithm can be programmed to read the difference between the values of the clicked pixels in all the bands and calculate statistically the parameters of the spectral distance in the different channels. The whole process could be made fully automatic but the user interaction and understanding is always fundamental. The application of this idea has been mainly dedicated to cartography but the algorithm is an object recognition tool that could work on any other multi-channel digital image.

\section{References}

[1] Soille, P.: Morphological Partitioning of Multispectral Images, Journal of Electronic Imaging 5(3) (July 1996) 252-265

[2] Ford, S.J., McKeown, D.: Utilization of Multispectral Imagery for Cartographic Feature Extraction, Proceedings of the DARPA Image Understanding Workshop, San Diego, CA. (Jan 1992) 805-820

[3] De Freitas, U.M., Câmara, G.: Developement of a Software for the Remote Sensing Community, Internal Report at D.P.I. (Divisão de Processamento de Imagem do I.N.P.E. Instituto Nacional de Pesquisas Espaciais), Brasil (1997)

[4] Kettig, R.L., Landgrebe, D.A.: Classification of Multispectral Data by Extraction and Classification of Homogeneous Objects, IEEE Transactions on Geoscience Electronics, Vol. GE-14, No.1 (Jan 1976) 19-26

[5] Cramariuc, B., Gabbouj, M. and Astola Jaakko, Clustering Based Region Growing Algorithm for Color Image Segmentation, Digital Signal Processing Proceedings, DSP'97, Vol.2 (July 1997) 857-860

[6] Beucher, S.: The Watershed Transformation Applied to Image Segmentation, $10^{\text {th }}$ Pfefferkorn Conf. On Signal and Image Processing in Microscopy and Microanalysis, Cambridge UK, Scanning Microscopy International (Sept. 1991). 
[7] Soille, P.: Morphological Image Analysis - Principles and Applications, Springer Verlag, Berlin (1998) 266-267

[8] Di Zenzo, S.: A Note on the Gradient of a Multi-image, Computer Vision, Graphics, and Image Processing 33 (1986) 116-125

[9] Cumani, A.: Edge Detection in Multispectral Images, Computer Vision, Graphics, and Image Processing: Graphical Models and Image Processing, 53(1) (1991) 40-51

[10] Beucher, S. : Segmentation d'Images et Morphologie Matematique, PhD thesis, Ecole de Mines de Paris (June 1990)

[11] Vincent, L., Soille, P.: Watershed On Digital Spaces: An Efficient Solution Based on Immersion Simulation, IEEE Transactions of Pattern Analysis and Machine Intelligence, vol.13, n.6, (1991) 593-598

[12] Soares, F., Muge, F.: Morphological Image Processing for Cartography, Cartografia e Cadastro, Instituto Português de Cartografia e Cadastro, No9, Portugal (1998) 39-49

[13] Barata, T., Pina, P.: Construction of Decision Region Borders by Geometric Modelling of Training Sets, Proceedings of ISMM 200, CSIRO Publishing, Sydney. (2002) 147-156. 